\title{
Development of a propulsion system for a biomimetic thruster
}

\author{
HA NgocSan ${ }^{1}$, GOO NamSeo ${ }^{1 *} \&$ YOON HyeonKyu ${ }^{2}$ \\ ${ }^{1}$ Department of Advanced Technology Fusion, Konkuk University, Seoul 143-701, South Korea; \\ ${ }^{2}$ Department of Naval Architecture \& Marine Engineering, Changwon National University, Gyeongnam 641-773, South Korea
}

Received July 9, 2009; accepted December 10, 2009

\begin{abstract}
We studied theoretically and experimentally a biomimetic propulsion system inspired by the motility mechanisms of bacteria such as E. coli. Our goal was to investigate the effect of the "complex" filament of Rhizobium Meliloti bacteria on thrust force. The complex filament is a helically perturbed filament, similar to a plain filament threaded through a small helix. The propulsive performance of this system was estimated by modeling the dynamics of helical wave motion in viscous fluid. The model consists of a helical filament which is axially rotated at angular velocity $\omega$. Resistive force theory (RFT) was applied to this model to calculate the thrust force and required torque. The Buckingham PI theorem (non-dimensional analysis) was also used to analyze the theoretical results. The procedure for making a complex filament with various pitch angles $\theta_{s}$ from a small helix and plain filament is explained in detail. To validate the theoretical results for helical wave propulsion and compare the characteristics of complex and plain filaments together, an experiment was performed to measure the thrust forces in silicone oil. The experimental results agreed with the theoretical values predicted by RFT. The thrust forces of complex filaments depended on the shape of small helix winding. The maximum thrust force was achieved at a small helix pitch angle of $\theta_{s}=45^{\circ}$. In addition, we found that the thrust force generated by a complex filament had a value about $10 \%$ higher than that of a plain filament with the same equivalent diameter $d_{e}$.
\end{abstract}

biomimetic micro-robots, swimming micro-robots, propulsion of flagella, medical application

Citation: Ha N S, Goo N S, Yoon H K. Development of a propulsion system for a biomimetic thruster. Chinese Sci Bull, 2011, 56: 432-438, doi: $10.1007 / \mathrm{s} 11434-010-4134-\mathrm{z}$

Recently, biomimetic micro-robots with high potential for medical applications have generated much interest. One of the ultimate goals of medical micro-robots is to transmit images from inside the human body, reach currently inaccessible areas, and carry out various complex operations $[1,2]$. Miniature, safe, and energy-efficient propulsion systems hold the key to developing this technology but they pose significant challenges. One of the challenges is propulsion. How micro-robots can operate inside the blood vessels of human body which are very narrow and full of highly viscous fluid has yet to be determined. Thus, novel motion methods are needed to adapt to this environment.

Many propulsion schemes have been proposed such as fin-driven, propellers or jet pumps. There are several types of biomimetic swimming robots, mostly based on fin, using

*Corresponding author (email: nsgoo@konkuk.ac.kr) shape memory alloys (SMAs), ionic exchange polymer metal composites (IPMCs), and most recently lightweight piezo-composite actuators (LIPCAs) [3-7]. Although most of these biomimetic robots are very effective on the macro-scale, miniaturization would make them ineffective because inertial forces become important for propulsion.

Accordingly, the microorganism approach is one of the best ways to imitate locomotion for the medical micro-robot field. Bacteria species such as Escherichia coli (E. coli) are favored to simulate the locomotion of minute filament. The cells of $E$. coli have one or more helical filaments, or flagella, which they use to swim. Each flagellum consists of a rotary motor that can rotate at about $100 \mathrm{~Hz}$ embedded in the cell wall, a short flexible hook functioning as a universal joint, and a helical filament $20 \mathrm{~nm}$ in diameter and $10 \mu \mathrm{m}$ in length. The helical filament can be considered to be a propeller that converts the rotary motion of the motor into lin- 
ear thrust [8]. For bacteria species, the Reynolds number $(R e)$ is about $10^{-4}$ wherein viscous effects are dominant over inertial effects. Therefore, the propulsion method of bacterial motion can perform effectively at low $R e$ numbers. Many studies have focused on the fundamental fluid mechanics surrounding locomotion from a simple helical filament attached to the cell body, and to the hydrodynamics of flagella bundling [8,9]. Recently, researchers investigated the fundamental fluid dynamics of a novel super-helical structure rotating in a Stokes fluid [10].

Until now, the shapes of flagella have not been experimentally widely studied. Although they are a most diverse species, bacteria have only two types of flagella filaments: "plain" and "complex". The complex filaments are helical forms of the plain ones [11]. The complex filaments have a rather coarse surface with deep grooves and ridges along right-handed or left-handed helical lines [11,12]. The surface view of a complex filament of Rhizobium lupine is shown in Figure 1. The number of helical lines depends on the type of flagella filaments. For instance, the complex flagella of Rhizobium meliloti 2011 has a diameter of 16 $\mathrm{nm}$, and their morphology is dominated by prominent undulations of an external three-start helix running at a $10 \mathrm{~nm}$ axial distance and at an angle of $32^{\circ}$ [13].

We focused on the potential of using flagella motion for micro-robot propulsion. We evaluated the effect of a "complex" filament on the thrust force produced by a single filament at low $R e$ numbers. In addition, the thrust forces of this motion were theoretically predicted using RTF (resistive force theory) theory. We simplified the complex filament with one small helix wound on a big helix called an "axial helix". Small helixes with different pitch angles were considered. We describe the construction of these "complex" filaments and "plain" filaments as well as the experimental setup. The experimental results show good agreement with the RFT result.

\section{Modeling of helical wave propulsion}

\subsection{Modeling}

A model was developed to evaluate a filament's thrust performance. Figure 2 shows the propulsion model. This dynamic model consists of a helical filament on one end which

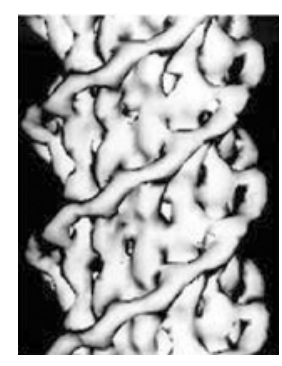

Figure 1 Surface view of flagella filament of Rhizobium lupine [12] rotates at an angular velocity of $\omega$. The other end moves freely in a fluid with viscosity $\mu$. We applied the RFT theory to predict the thrust force and the required torque of the motor. In RFT theory, the normal and tangential components of drag force $\mathrm{d} F_{n}$ and $\mathrm{d} F_{s}$ on each element of the slender body are proportional to the respective components of the local velocity with different proportional constants in a region where the $R e$ number is less than 1 [14]. Thus,

$$
\begin{aligned}
& \mathrm{d} F_{n}=-C_{n} V_{n} \mathrm{~d} s \\
& \mathrm{~d} F_{s}=-C_{s} V_{s} \mathrm{~d} s
\end{aligned}
$$

where $V_{n}$ and $V_{s}$ are the normal and tangential velocities of the element, respectively, and $C_{n}$ and $C_{s}$ are the corresponding coefficients of resistance, given by Brennen and Winet $[15]$ as

$$
\begin{gathered}
C_{n}=\frac{4 \pi \mu}{\ln \frac{2 \lambda}{a}+\frac{1}{2}}, \\
C_{s}=\frac{2 \pi \mu}{\ln \frac{2 \lambda}{a}-\frac{1}{2}},
\end{gathered}
$$

where $\lambda$ and $a$ are the wavelength and the radius of the filament, respectively.

When the motor rotates at a constant speed, every crosssection of the filament rotates about the $z$-axis with an anglular velocity $\omega$. Thus, the velocity in the $\theta$ direction is $V_{\theta}=\omega A$. The normal and tangential velocity components in the normal and tangential directions at the $\mathrm{d} s$ component are $V_{n}=V_{\theta} \sin \theta$ and $V_{s}=V_{\theta} \cos \theta$.

From the free body diagram of the forces acting on element $\mathrm{d} s$ of the tail, the propulsive force in the $z$-direction, in terms of its normal and longitudinal components, is

$$
\mathrm{d} F_{z}=\mathrm{d} F_{s} \sin \theta-\mathrm{d} F_{n} \cos \theta
$$

The force in the $\theta$-direction and the torque created by this force are defined as

$$
\begin{gathered}
\mathrm{d} F_{\theta}=-\left(\mathrm{d} F_{s} \cos \theta+\mathrm{d} F_{n} \sin \theta\right), \\
\mathrm{d} M_{z}=-A\left(\mathrm{~d} F_{s} \cos \theta+\mathrm{d} F_{n} \sin \theta\right),
\end{gathered}
$$

where $A$ is the amplitude of the helix and $\theta$ is the constant pitch angle between the tail and the perpendicular axis to the tail, which have a relation:

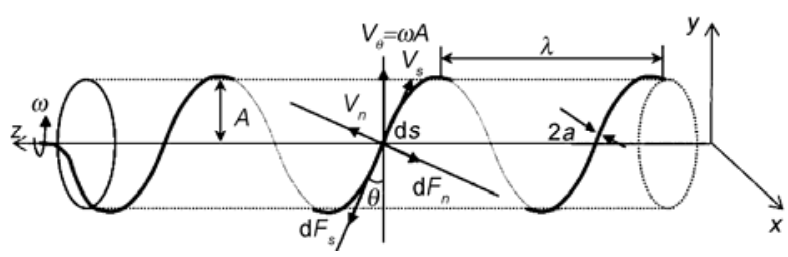

Figure 2 Propulsion model of a filament. 


$$
\tan \theta=\frac{\lambda}{2 \pi A}
$$

Assuming that all the infinitesimals of the tails are in the same moving situation, and if the number of helical waves along the tail is $n$, then, the thrust force can be written as

$$
\begin{aligned}
F_{z} & =\int_{0}^{n \lambda} \mathrm{d} F_{z}=\int_{0}^{n \lambda}\left(\mathrm{d} F_{s} \sin \theta-\mathrm{d} F_{n} \cos \theta\right) \\
& =V_{\theta} \sin \theta \cos \theta \int_{0}^{n \lambda}\left(C_{n}-C_{s}\right) \mathrm{d} s .
\end{aligned}
$$

The length of the tail $\mathrm{d} s$ can be written as

$$
\begin{aligned}
\mathrm{d} s & =\frac{\mathrm{d} x}{\sin \theta}, \\
F_{z} & =V_{\theta} \cos \theta \sin \theta \int_{0}^{n \lambda}\left(C_{n}-C_{s}\right) \frac{\mathrm{d} x}{\sin \theta} \\
& =n \lambda \omega A \cos \theta\left(C_{n}-C_{s}\right) .
\end{aligned}
$$

The required torque of the motor can be written as

$$
\begin{aligned}
T_{z} & =\int_{0}^{n \lambda} \mathrm{d} M_{z}=\int_{0}^{n \lambda}-A\left(\mathrm{~d} F_{s} \cos \theta+\mathrm{d} F_{n} \sin \theta\right) \\
& =\int_{0}^{n \lambda} A\left(C_{s} \omega A \cos ^{2} \theta+C_{n} \omega A \sin ^{2} \theta\right) \mathrm{d} s \\
& =\omega A^{2} \int_{0}^{n \lambda}\left(C_{s} \cos ^{2} \theta+C_{n} \sin ^{2} \theta\right) \frac{\mathrm{d} x}{\sin \theta} \\
& =n \lambda \omega A^{2} \frac{\left(C_{s} \cos ^{2} \theta+C_{n} \sin ^{2} \theta\right)}{\sin \theta} .
\end{aligned}
$$

Buchingham PI theorem [16] (non-dimensional analysis) of the force and the required torque of this system show that

$$
\begin{aligned}
\frac{F}{\mu \omega L^{2}} & =f_{F}\left(\frac{A}{L}, \frac{a}{L}, \frac{\lambda}{L}\right), \\
\frac{M}{\mu \omega L^{3}} & =f_{M}\left(\frac{A}{L}, \frac{a}{L}, \frac{\lambda}{L}\right) .
\end{aligned}
$$

From eqs. (11) and (12), we conclude that both the thrust force and required torque of the motor are functions of the geometry of flagellum only.

\subsection{Theoretical dynamic performance analysis}

Our goal is to optimize the pitch angle of the helix so that it produces the maximum thrust force, if the length and amplitude of the helix are given. Figure 3 shows the values of thrust force with several pitch angles $\theta$ for angular velocity $\omega$. In this case, the length and diameter of the filament are $72 \mathrm{~mm}$ and $0.88 \mathrm{~mm}$, respectively. The amplitude of the helix is $2.5 \mathrm{~mm}$. The optimum value of pitch angle is around $40^{\circ}$. To obtain the exact peak of the force in the region from $35^{\circ}$ to $45^{\circ}$, we divided this region in steps of $1^{\circ}$. In this way, we obtained the maximum thrust force produced by plain filament at a pitch angle of $\theta=42^{\circ}$.

From our non-dimensional analysis, the non-dimensional force and required torque versus the amplitude-to-length ratio $A / L$ depended on pitch angle $\theta$ and the $\lambda / A$ ratio. The experimental observations revealed that filaments with $L$ $>>a$ satisfied slender body condition [9]. Hence, $l=L / a(l$ is the ratio of the total length of the flagellum in the direction of propulsion to its radius) is on the order of $O(100)$ [9]. The theoretical analysis of non-dimensional force and the required torque against $A / L$ for several given values of $\theta$ and $l$ is shown in Figure 4, where $l$ is taken to be 200. For example, at $\theta=45^{\circ}$, as the ratio $A / L$ increases, both the thrust force and the required torque of the motor will increase. When the $A / L$ ratio increased from zero to a certain value, the thrust force increased dramatically while the increment of required torque remained small. Hence, the suitable value of $A / L$ lies in the range somewhere between 0 and 0.03 . This predicted value of $A / L$ is fairly well supported by observed data (0.025). From observation, E. coli bacteria were propelled by rotating a left-handed helical filament whose diameter and length were approximately 20 $\mathrm{nm}$ and $10 \mu \mathrm{m}$, respectively. This helical filament had a wavelength of about $2.5 \mu \mathrm{m}$ and a helix diameter of about $0.5 \mu \mathrm{m}$ [17]. Thus, the ratio of $A / L$ for a typical $E$. coli bacterium was about 0.025 . For $\theta=60^{\circ}$, the non-dimensional force and torque did not change significantly compared with the case of $\theta=45^{\circ}$. Thus, the $A / L$ was chosen to be the same as in the case of $\theta=45^{\circ}$. We note that for fixed $L, A / L$ should be suitably chosen for each case of pitch angle $\theta$.

The non-dimensional force and required torque were plotted against the radius-to-length ratio $a / L$ in Figure 5

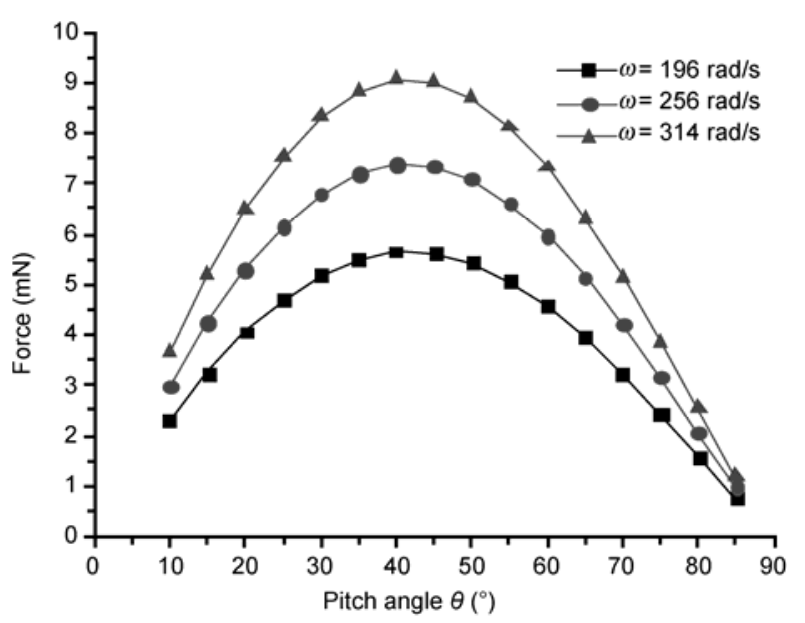

Figure 3 Thrust force versus pitch angle for various values of angular velocity of the filament. 


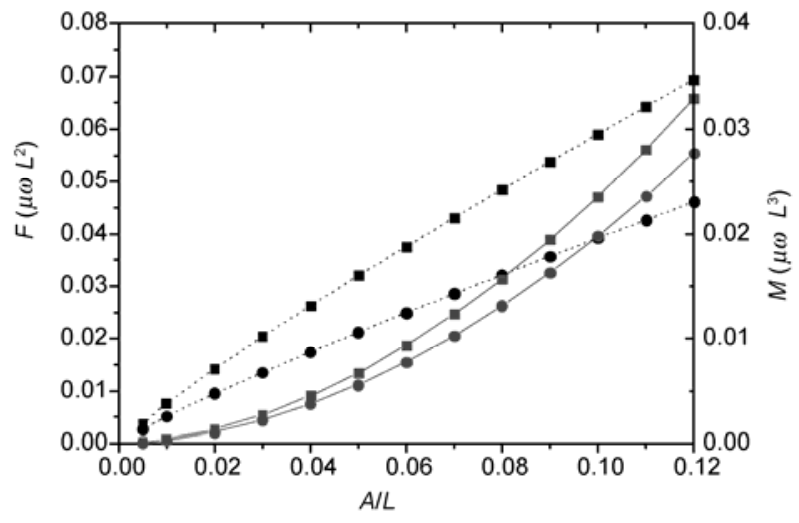

Figure 4 The non-dimensional thrust force and the required torque of the motor versus $A / L$ ratio with various pitch angles $\theta \quad\left(\theta=45^{\circ}(\boldsymbol{\bullet}), \theta=60^{\circ}\right.$ $(\bullet))$.

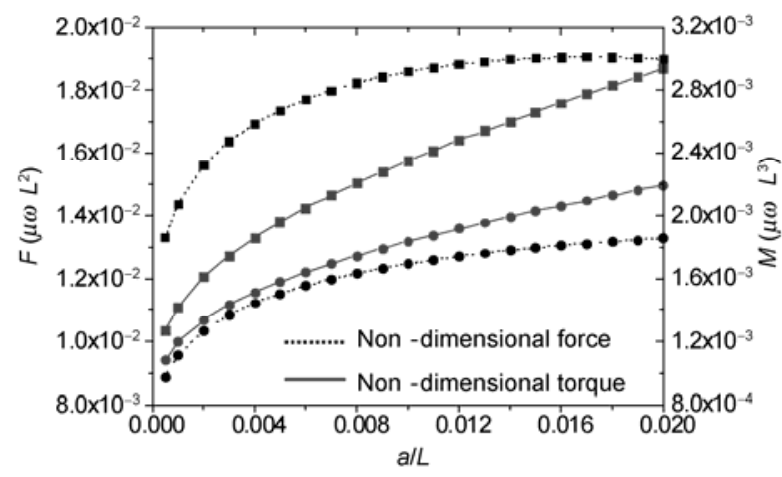

Figure 5 Non-dimensional thrust force and the required torque of the motor versus $a / L$ ratio with various pitch angles $\theta\left(\theta=45^{\circ}(\mathbf{\bullet}), \theta=60^{\circ}\right.$ $(\bullet))$.

with a fixed $A / L=0.025$ and various pitch angles $\theta$. The graph in this figure shows that as the ratio of $a / L$ increased, both the non-dimensional force and required torque increased. When the ratio of $a / L$ increased to a certain value (0.004), the dimensionless force increased dramatically. Beyond that range, the dimensionless force did not increase so much. Our observation of bacteria flagella suggests that the $a / L$ ratios are very small. For instance, at $A / L=0.025$ and $\theta=60^{\circ}$, the value of $a / L$ for a typical bacterium is 0.001 , which can be found in [17].

Figure 6 shows that both the non-dimensional thrust force and the required torque of the motor are functions of the $\lambda / L$ ratio with fixed $A / L=0.025$ and various $a / L$ ratio. The graphs show that as the ratio of $\lambda L$ increased, both the non-dimensional thrust force and the required torque decreased. When the ratio of $\lambda / L$ increased to a certain value $(0.3)$, the dimensionless force and required torque decreased dramatically. Beyond that range, the dimensionless required torque converged to a certain value while the dimensionless force still decreased. Hence, the suitable value of $\lambda / L$ is about 0.3 . This predicted value of $\lambda L$ is fairly well supported by observed data (0.25) [17].

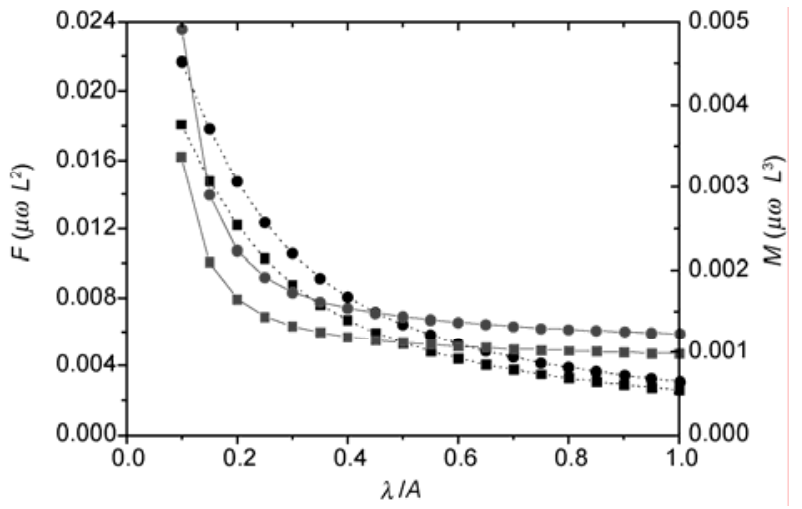

Figure 6 The non-dimensional thrust force and the required torque of the motor versus $\lambda / L$ ratio with various ratios of $a / L(a / L=0.001(\square), a / L=$ $0.005(\bullet))$.

\section{Filament fabrication and experiment}

\subsection{Filament fabrication}

A complex filament was made from an aluminum wire. The wire was sufficiently malleable to be formed into the desired shape, and rigid enough to be undeformed as it rotates in the viscous fluid. The complex filament was made in two steps shown in Figure 7.

First, a small aluminum wire (diameter $0.15 \mathrm{~mm}$ ) was tightly bent in a counterclockwise direction around an aluminum rod (diameter $=0.5 \mathrm{~mm}$ ). The axial helix was made using the same procedure, but we used a bigger diameter wire (diameter $=0.5 \mathrm{~mm}$ ) bent along the helical path on the $5 \mathrm{~mm}$ diameter rod. The important difference between the two helices is their twisting direction; the axial helix was bent in a counterclockwise direction, whereas the small helix was bent in a clockwise direction. The axial helix was threaded through the small helix, forming a complex fila-

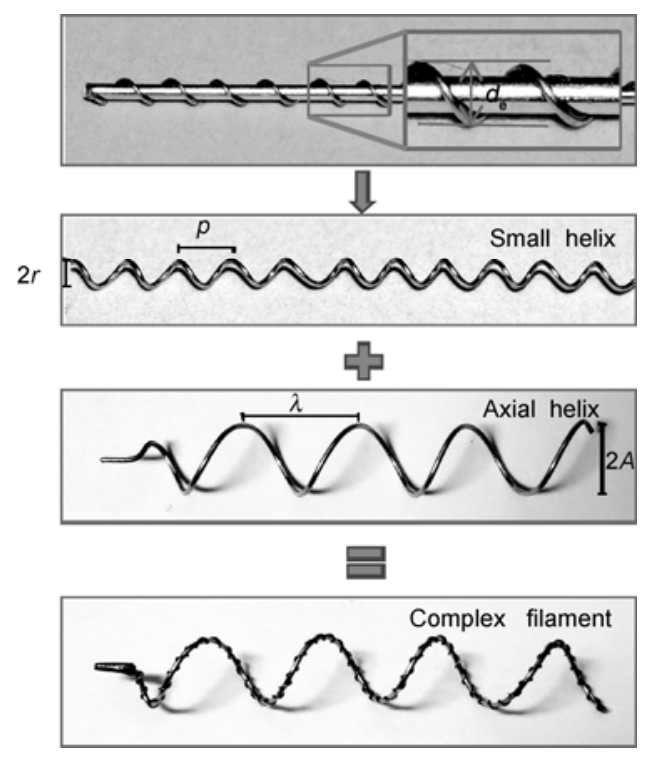

Figure 7 Procedure for making complex filament. 
ment [10]. The plain filament was made following the same procedure as the axial helix. A schematic of plain and complex filaments is shown in Figure 8 and their dimensions are given in Table 1.

The defined geometric parameters of the complex filament are the radius $r$ and the pitch $p$ of the small helix, the radius $A$ and the pitch $\lambda$ of the axial helix, as shown in Figure 7 . We fixed the geometry of the axial helix and changed the geometry of the small helix. The pitch angles $\theta_{s}$ of the small helix were verified from $30^{\circ}$ to $60^{\circ}$ with each step forward of $5^{\circ}$. Seven complex filaments with different small helices are shown in Figure 9.

\subsection{Experiment}

A schematic of the experimental setup is shown in Figure 10. A direct current (DC) motor (12 $\mathrm{mm}$ in diameter and $20 \mathrm{~mm}$ in length) was located at the head of the swimmer prototype and fixed at a supporter. The supporter is made of acrylic and has a hole whose diameter is the same as the motor's diameter. The motor was fixed to the supporter simply by sliding it into place. The supporter was connected to a load cell (Nano17, ATI Industrial Automation, resonant frequency $7.2 \mathrm{kHz}$, resolution $1 / 1280 \mathrm{~N}$ ) which we used to measure the thrust force. The filament was vertically connected to the motor through a coupling. The stationary motor rotated the filament immerged in a tank of silicone oil (Shinetsu Co.). The viscosity of the silicone oil was $350 \mathrm{cst}$, which is 350 times higher than the viscosity of the water. The silicone container was $320 \mathrm{~mm}$ in length, $200 \mathrm{~mm}$ in depth and $190 \mathrm{~mm}$ in width. We ensured that the tail was fully submerged in the oil and sufficiently far from the wall and the bottom of the container. The motor operated at voltages from $0.4 \mathrm{~V}$ to $1.2 \mathrm{~V}$ with steps of $0.2 \mathrm{~V}$. The resulting frequency of rotation was measured with a highspeed camera (APX Ultimate, Japan) operating at 1000 frames/s to capture the rotation motions.

To compare the thrust force of each filament, an experimental setup was constructed to measure the thrust force at Reynolds numbers smaller than 1 . To simplify the fabrication process, the dimension of the tail was scaled up according to the Buckingham PI theorem [16]. This method confirms the ratio of $F /\left(\mu \omega L^{2}\right)$ is the same for the scaled up filament and the micro-scale filament. The dimensions of flagella are given in Table 1.

\section{Results and discussion}

Figure 11 depicts the experimental results for the thrust force generated by the helical wave propulsion of the plain filament given in Table 1. The experiment was performed in silicone oil. As predicted by theory, the experimental values of force varied linearly with the angular velocity of rotation for the plain filament. The critical factor in this experiment was wall effect. To reduce the wall effect, the walls of the container must be designed far enough from the filament so that the wall will not affect the fluid flow significantly. It was shown that below $R e=1$, no wall effect condition will

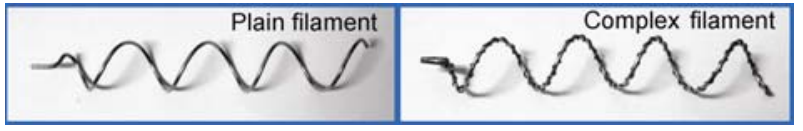

Figure 8 Schematic of plain and complex filaments.

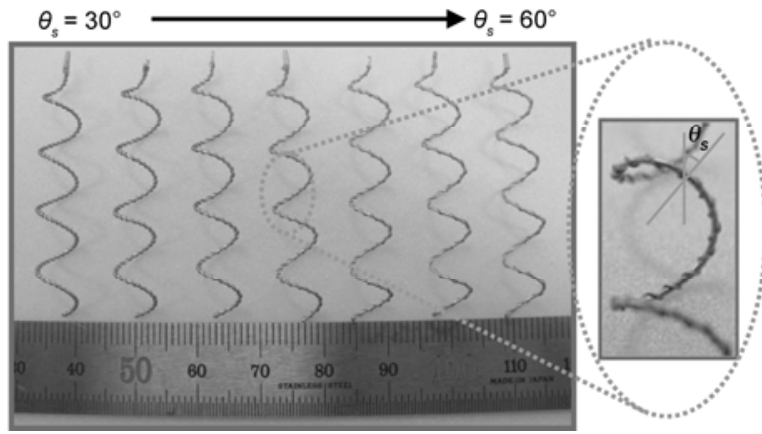

Figure 9 Seven complex filaments with different pitch angles of the small helix.
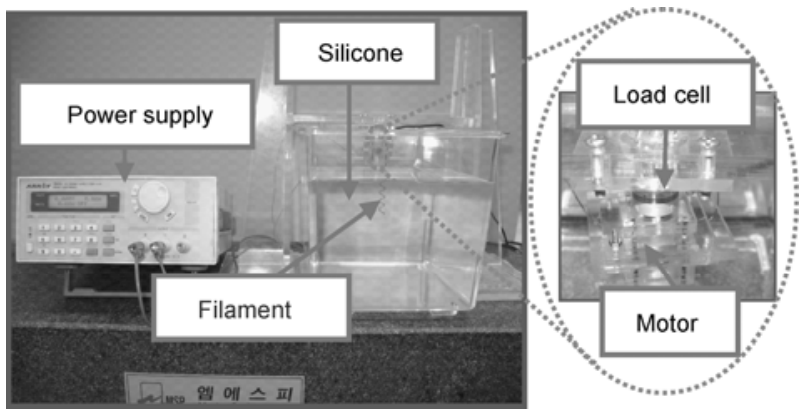

Figure 10 Schematic of thrust force measurement system.

Table 1 Dimensions of filaments

\begin{tabular}{|c|c|c|c|}
\hline \multirow{2}{*}{ Dimension } & \multicolumn{2}{|c|}{ Complex filament } & \multirow{2}{*}{ Plain filament } \\
\hline & Small helix & Axial helix & \\
\hline Amplitude of helix $A$ & $0.44 \mathrm{~mm}$ & $2.5 \mathrm{~mm}$ & $2.5 \mathrm{~mm}$ \\
\hline Wavelength $\lambda$ & Depend on $\theta_{\mathrm{s}}$ & $9 \mathrm{~mm}$ & $9 \mathrm{~mm}$ \\
\hline Length $L$ & Depend on axial helix & $36 \mathrm{~mm}$ & $36 \mathrm{~mm}$ \\
\hline
\end{tabular}


be achieved if the distance satisfies $y>(20 l / R e)$ away from the body in which $L$ is the characteristic length of the body [2]. In this experiment, since the condition of $y>(20 l / R e)$ was satisfied, the wall effect can be ignored.

The thrust forces of complex filaments versus the angular velocity of rotation are shown in Figure 12. As in the plain filament case, the experimental thrust forces are linear with respect to the angular velocity of rotation. The thrust forces produced by complex filaments are higher than forces from plain filaments (complex filaments without small helix). This fact can be explained by the RFT theoretical predictions. For this experiment, the complex filaments without the small helix had the ratio $a / L=0.007$ whereas the complex filaments had an equivalent diameter $d_{\mathrm{e}}=0.8 \mathrm{~mm}$ as defined in Figure 7, and the ratio $a / L=0.011$, respectively. Pitch angle $\theta=30^{\circ}$ and the ratio $A / L=0.07$ were set the same for both of complex filaments and complex ones without small helix. We calculated the thrust force using eq. (11) for $\theta=30^{\circ}$ and $A / L=0.07$. The calculated non-dimensional thrust forces are 0.0615 for the complex filament and 0.0586 for the plain filament. In conclusion, the complex filament produced a higher thrust force than the filament without the small helix.

In Figure 12 we note that the complex filament for $\theta_{s}=$ $45^{\circ}$ produced a higher thrust force than the plain one with the same equivalent diameter $(0.8 \mathrm{~mm})$. For an applied voltage from $0.6 \mathrm{~V}$ to $1 \mathrm{~V}$ the thrust forces increased by 6 to 20 percent.

The effect of the small helix on the propulsion produced by complex filaments is shown in Figure 12. The maximum thrust force in this experiment for a set of complex filaments was achieved at a pitch angle of small helix $\theta_{\mathrm{s}}=45^{\circ}$. In this study, since we considered the pitch angle $\theta_{\mathrm{s}}$ from

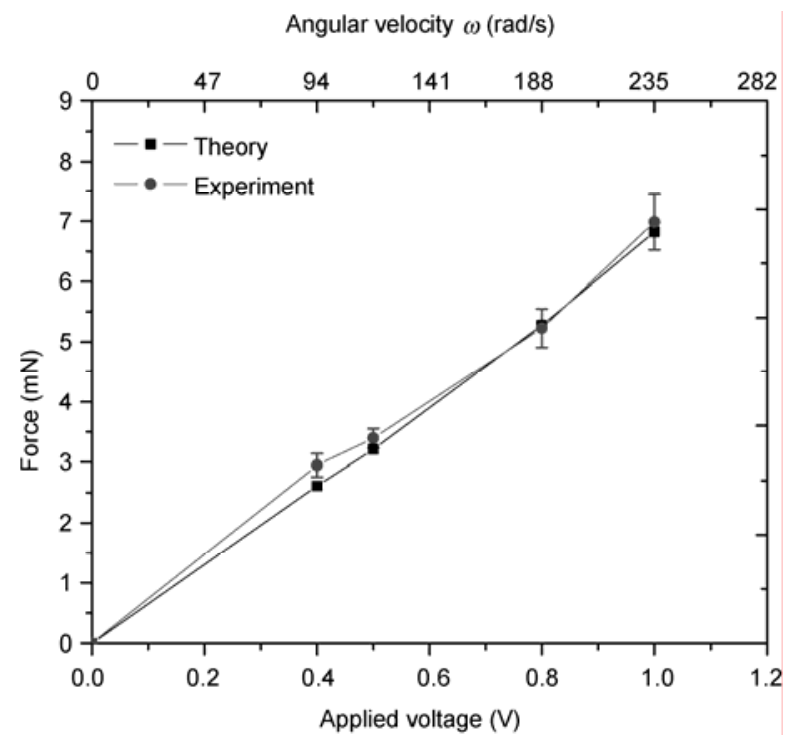

Figure 11 Experimental result for the thrust force created by plain filament given in Table 1.

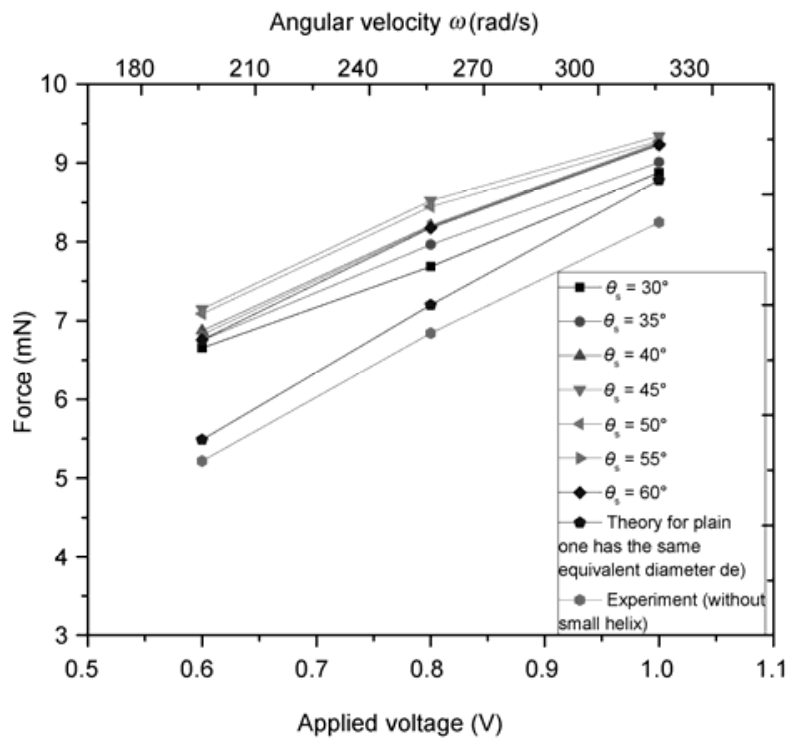

Figure 12 Experimental result for the thrust force created by plain flagellum given in Table 1.

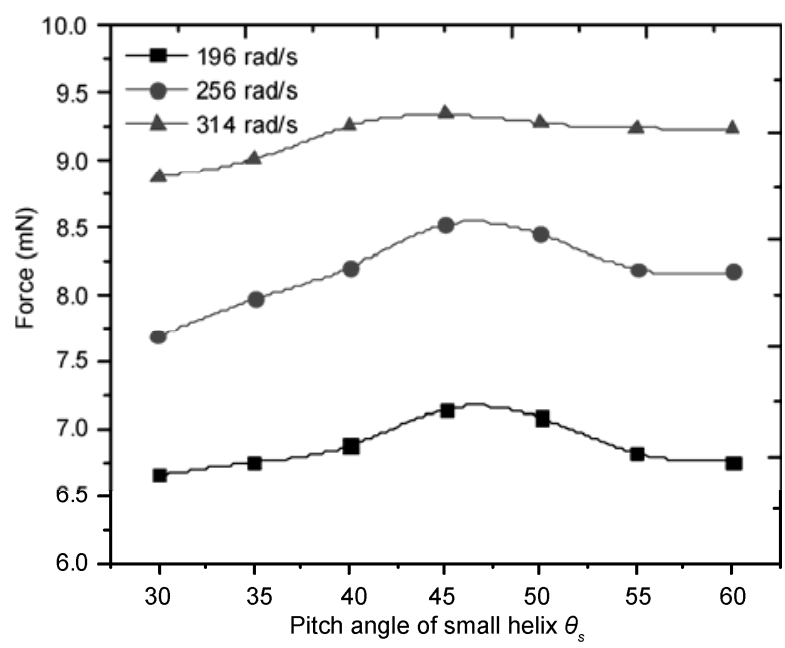

Figure 13 Experimental result for the thrust force of complex flagellum with various pitch angles of small helix.

$30^{\circ}$ to $60^{\circ}$ with a step of $5^{\circ}$, we can predict it will exist between $40^{\circ}$ and $50^{\circ}$. We found that the thrust forces became constants beyond $55^{\circ}$ of pitch angle, which means that the small helix did not increase the thrust force for the large pitch angle. The optimum value of $\theta_{\mathrm{s}}$ was around $45^{\circ}$.

When we compare Figures 3 and 13, the pitch angles for maximum force are similar. This means the analytic and experimental results are in good agreement.

\section{Conclusions}

A biomimetic thruster based on the motion of bacteria was studied theoretically and experimentally. A dynamic model of the swimming method was developed to predict the val- 
ues for thrust force created by the helical flagellum and the required torque of the driving motor. By applying the Buckingham PI theorem, a scaled-up model of a flagellum was constructed and tested in silicone oil. For the plain flagellum, the experimental results were shown to be in good agreement with theoretical values predicted by the RFT model. The effect of the complex filament on propulsion was considered. The thrust force created by complex filament was higher than the complex filament without the small helix. The maximum thrust force of the complex flagellum was achieved at a pitch angle of the small helix of $\theta_{\mathrm{s}}$ $=45^{\circ}$. We found that the thrust force generated by the complex flagellum had a higher value (by about 10 percent) than the plain flagellum with the same equivalent diameter.

This work was supported by Konkuk University in 2010. The authors are grateful for the financial support.

1 Bogue R. The development of medical microrobots: A review of progress. J Ind Robot, 2008, 35: 294-299

2 Sitti M. Design methodology for biomimetic propulsion of miniature swimming robots. J Dyn Syst Meas Contr, 2006, 128: 36-43

3 Wang Z, Hang G, Li J, et al. A micro robot fish with embedded SMA wire actuated flexible biomimetic fin. J Sens Actuators, 2008, 144: $354-360$

4 Kim B, Kim D H, Jung J, et al. A biomimetic undulatory tadpole robot using ionic polymer-metal composite actuators. Smart Mater
Struct, 2005, 14: 1579-1585

5 Fukuda T, Kawamoto A, Arai F, et al. Steering mechanism of underwater micro mobile robot. In: Proc. IEEE Int Conf on Robo and Auto Nagoya, Japan, 21-27 May 1995. 363-368

6 Zhang W, Guo S X, Asaka K J. A new type of hybrid fish-like microrobot. Int J Autom Comput, 2006, 3: 358

7 Nguyen Q S, Heo S, Park H C, et al. A fish robot driven by piezoceramic actuators and a miniaturized power supply. Int $\mathrm{J}$ Control Autom Syst, 2009, 7: 267-272

8 Kim M J, Bird J C, van Parys A J, et al. A macroscopic scale model of bacterial flagellar bundling. Proc Nat Acad Sci USA, 2003, 100: 15481

9 Chwang T, Wu T. A note on the helical movement of micro-organisms. Proc R Soc London Ser B, 1971, 178: 327-346

10 Jung S H, Mareck K, Fauci L, et al. Rotational dynamics of a superhelix towed in a stokes fluid. Phys Fluids, 2007, 19: 103105

11 Schmitt R R, Raska I, Mayer F. Plain and complex flagella of Pseudomonas rhodos: Analysis of fine structure and composition. J Bacteriol, 1974, 117: 145-162

12 Trachtenberg S, Fishelov D, Ben-Artzi M. Bacteria flagellar microhydrodynamics: Laminar flow over complex flagellar filaments, ano$\log$ archimedean screws and cylinders and its perturbations. Biophys J, 2003, 85: 1345-1357

13 Krupski G, Gotz R, Ober K, et al. Structure of complex flagellar filaments in Rhizobium meliloti. J Bacteriol, 1985, 162: 361-366

14 Hancock G. The self-propulsion of microscopic organisms through liquids. Proc R Soc London Ser A, 1953, 217: 96-121

15 Brennen $\mathrm{C}$, Winet $\mathrm{H}$. Fluid mechanics of propulsion by cilia and flagella. Annu Rev Fluid Mech, 1977, 9: 339-398

16 Fox R, McDonald A, Pritchard P. Introduction to Fluid Mechanics. New York:Wiley, 2004

17 Howard C. Symmetries in bacterial motility. Proc Natl Acad Sci USA, 1996, 93: 14225-14228

Open Access This article is distributed under the terms of the Creative Commons Attribution License which permits any use, distribution, and reproduction in any medium, provided the original author(s) and source are credited. 